\title{
Research on Cooling Efficiency Evaluation of Split Type Cooling Transformer based on ADC
}

\author{
Bengang Wei ${ }^{1,2, a,{ }^{*}, \text { Junshang Lou }}{ }^{3, b}$, Hua Huang ${ }^{1, c}$, Jie Lou ${ }^{4, d}$, \\ and Yuying Zhang ${ }^{4, d}$ \\ ${ }^{1}$ Shanghai Electric Power Research Institute, State Grid, Handan Road, Shanghai, China \\ ${ }^{2}$ Shanghai SEPRI Power Technology Co., LTD., Song hua jiang Road, Shanghai, China \\ ${ }^{3}$ Shanghai Municipal Electric Power Company, State Grid, Yuan shen Road, Shanghai, China \\ ${ }^{4}$ School of Electrical Engineering, Shandong University, Jing shi Road, Jinan, China \\ awbgsj@126.com, b16567583@qq.com, chuahuang@sinan.com, ddawn.zyy@gmail.com
}

Keywords: The split type cooling transformer, cooling efficiency evaluation, ADC, AHP.

\begin{abstract}
This paper proposed an efficiency evaluation model for cooling system of the split type transformer using ADC (availability dependability capacity) method. This proposal is presented based on the principles and the sophisticated characteristics of the split type cooling transformer. Data acquisitions of system operations, technologies, usage rules and cooling effects, comparing with acquirable data for calculation from the split cooling transformer manufacturer is included of this model proposal as well. In the proposed model, the enhanced AHP method is used for identifying the core capability matrix and for further calculation of the efficiency quantities. This proposal is verified through an example of cooling efficiency evaluating of the cooling system of a $220 \mathrm{kV}$ oil-water transformer. The testing results of this example proved that the proposed model can accurately and effectively evaluate the cooling efficiency of the cooling system of the split transformer.
\end{abstract}

\section{Introduction}

Due to the rapid growing of the electricity loads in city areas, the number of substation constructions in these areas is increasing as well. Requirements of coordination land usages and environment issues in these cities, especially large cities, such as Being and Shanghai, leads to the increasing of the newly built underground and indoor substations with voltage levels over $110 \mathrm{kV}$. Due to the limitation of space and the cooling requirement of power equipment, the cooling system of these underground and indoor transformer substations is usually separated from the transformers [1-3]. At present, many scientific research institutes have established technical evaluation systems of power equipment conditions. However, the focus is still mainly on the operation states of transformer. So far, there has not been a mature evaluation system for the cooling system - auxiliary equipment of the split type cooling transformer which has gradually increased over the recent years.

The cooling modes and system structure of the split type cooling transformer are complex and diverse. Classifying from different internal oil circulation modes, the oil immersed transformer can be divided into natural circulation type, forced circulation type and strong oil guiding type. Classifying from different cooling methods of the external radiator, the transformer types can be divided into natural air cooling, forced air cooling and forced water cooling. The cooling system includes water cooler, circulating water pumps, cooling towers, heat exchangers, circulating oil pumps, heat sinks, etc. ${ }^{[3]}$. Due to the obvious complexity of the transformer's structure and its cooling method, the defect classification of cooling system, the simulation and the calculations of cooling can be very sophisticated. Therefore, it is quite difficult to evaluate the effectiveness of split type cooling system and also, as the split type cooling system plays a key role in safely and reliably operating of the transformer, its performance evaluation is particularly of crucial importance. Therefore, the evaluation of the efficiency of the transformer's cooling system has meanings to the guidance, 
diagnosis, management and identification of the usages and managements of transformer cooling efficiency system and the operation and maintenance of transformer.

In this paper, the evaluation of the split cooling system's efficiency is realized through an established efficiency evaluation system. This system establishment is based on the calculation of the heat simulation model, system operations and maintenances analysis model and using a worldwide common method, the ADC method. With the acquisition of the specified time and operational conditions, the ability and quality of the cooling process within a transformer cooling system can be identified. This paper presents the content of evaluation criteria with the considerations of the characteristics of the cooling system, proposes quantity evaluation based on ADC method and identifies capacity matrix using AHP method. The proposed model is verified through an example of a $220 \mathrm{kV}$ transformer in reality and the results show good practicability.

\section{Establishment of Efficiency Evaluation Criteria System}

The operation efficiency of the cooling system is influenced by many factors. These factors include the cooler efficiency, equipment operation status, surroundings and operation environment, loads of the equipment, the aging level of the equipment, outer pollutant, etc. Therefore, the established evaluation system should be an overall system to comprehensively consider all the scenarios. As the efficiency of the split system has close correlations with the transformer's heating temperature, temperature distribution characteristics, thus, the capability of the split cooling system can be described by these two criteria. In the above descriptions, many factors have played roles in the output variable of the split transformer cooling system, and the efficiency of this system is mainly evaluated through the cooling effect that is performed to the transformer. Therefore, the evaluation system should first focus on the suitability of the cooling system and the transformer and the standard for this system for evaluation should be a multi-dimension evaluation system that considers all sorts of information.

As stated above, three criteria should be included in the evaluation system. The first one is a quantifying system to overall reflect the split type cooling system's characteristics and status, or also called the criteria system. The second one is influential directions of all the criteria in the criteria system to the overall efficiency of the cooling system, or also called the contribution influential direction. The third one is contribution proportion of all the criteria in the criteria system to the overall efficiency of the cooling system.

The criteria system of evaluating the split transformer cooling system mentioned above consists of the content of the following four aspects,

1) Criteria reflecting operational characteristics of the cooling system itself. These characteristics include equipment defection, malfunctions, component aging, etc.

2) Criteria reflecting adaptability of the cooling equipment, which is optimization of the cooling methods. For instance, the oil immersed transformer can be divided into natural circulation type, forced circulation type and strong oil guiding type, etc.

3) Criteria reflecting operation rules, such as system starting coefficients, auxiliary heat dissipation.

4) Criteria reflecting the ultimate cooling effects, such as transformer top layer oil temperature increment, transformer heating points temperature increment, etc.

Besides these aspects, this criteria system should also reflect initial cooling effect of the cooling system. This mainly considers the trial and operational data from the manufacturer of the equipment.

\section{Split Cooling System Efficiency Evaluation System}

ADC method is one of the classical methods in evaluating equipment efficiency. Availability, dependability and Capacity are used to evaluate Effectiveness of the equipment. These three variables can be combined to express one quantifying factor which demonstrates the overall efficiency performance of the entire system. The expression is written as below,

$\mathrm{E}=\mathrm{ADC}$ 
The efficiency of the split cooling system can be described by many different criteria, and each criterion is a quality coefficient, the performances of these coefficients can represent the overall efficiency of the cooling system. The quality coefficients are usually represented through possibilities, and the quality and quantity of the quality coefficients are largely dependent based on the factors of the specific experiment tasks which the transformer is targeting. Once the operation environment and conditions are certain, the efficiency of the transformer can be identified as well. Therefore, the definition and algorithms of ADC method is effective in quantifying the efficiency of the split transformer cooling system. With certain operation status and modes, ADC method can make the model simpler by quantifying all criteria relating to the efficiency. As on-line monitoring and other monitoring techniques develop, the using of all-around data from these monitoring and the combination with ADC method can evaluate the efficiency of the cooling system in a more accurate way.

\subsection{Analysis of the Evaluation Criteria of Split Cooling System}

Probability A is used to indicate the possibility of one status which might occur at a random spot in the cooling system, and it is determined by the reliability and maintenance of the equipment. The split cooling system has only in service and out of service modes ${ }^{[6]}$,

$$
\begin{aligned}
& \mathrm{A}=\left[\mathrm{a}_{1}, \mathrm{a}_{2}\right] \\
& \mathrm{a}_{1}=\frac{M T B F}{M T B F+M T T R} \\
& a_{2}=1-a_{1}
\end{aligned}
$$

Where is the possibility of the cooling system in service, is the possibility of the cooling system out of service, is the average service time between two outages, expressed by, is the operation time before the time outage, $\mathrm{n}$ is the time between outages, is the average recovering time from outages, expressed in , is the recovery time from the outages in the time outage, $n$ is the times of outages.

Credibility D is a matrix representing the possibility which certain quantities reach certain status. These quantities represent status of any location within the cooling system during operations. The credibility of the split cooling system is determined by maintenance, reliability and other factors. As split cooling system has only in service and out of service modes, the credibility matrix can be expressed as,

$$
\mathrm{D}=\left[\begin{array}{l}
d_{11} d_{12} \\
d_{21} d_{22}
\end{array}\right]
$$

In the expression, represent the possibility of cooling system in service and then out of service. Represent the possibility of cooling system out of service and then in service after reparation. For split cooling system, when not in operational status when put into operation, then the system will not be repaired during operation. Therefore,

$$
\mathrm{D}=\left[\begin{array}{l}
d_{11} d_{12} \\
d_{21} d_{22}
\end{array}\right]=\left[\begin{array}{cc}
\exp \left(\frac{-t}{M T B F}\right) & 1-\exp \left(\frac{-t}{M T B F}\right) \\
0 & 1
\end{array}\right]
$$

Capability $\mathrm{C}$ is the quantity for completing certain task when the status of the cooling system is known. This capability is represented through all sorts of characteristics of the cooling system. Therefore, the capability matrix should be established based on the specific task which the transformer is undertaking. The objective of the cooling system is to realize the heat dissipation of the transformer, and this objective is mainly monitored through the suitability, reliability, adaptability, etc. Capability is the core for evaluating the efficiency of the cooling system, due to its complex structure, large number of influential factor which are hard to quantify, the objective to quantify the capability is the core issue in investigating the efficiency of the cooling system. The next subsection uses AHP methods to establish a multi-dimension capability vector for the solution of the efficiency evaluation.

\subsection{Structural Diagram of the Cooling System}

The factors which would affect the capability of the cooling system are listed as blow,

1) Operational criteria, these are vital factors which include equipment malfunction, defections and component aging. 
2) Adaptability, which is optimization of the cooling methods. For instance, the oil immersed transformer can be divided into natural circulation type, forced circulation type and strong oil guiding type, etc.

3) Operation rules, such as system starting coefficients, auxiliary heat dissipation.

4) Ultimate cooling effects, such as transformer top layer oil temperature increment, transformer heating points temperature increment, etc.

\subsection{Capability Model of Cooling System}

The structure diagram is shown in Figure 1 .

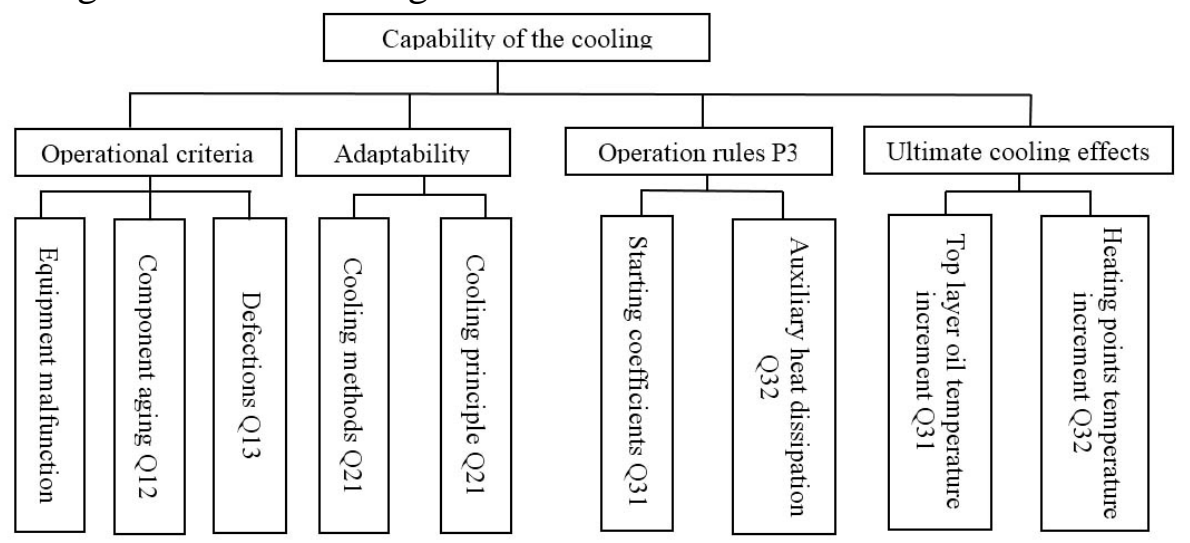

Figure 1. Hierarchical Structure of the Capability of the Cooling System

Decision matrix from $\mathrm{Q}$ to $\mathrm{P}$ can be expressed as the following:

Decision matrix from Q1 to P1 can be expressed as $\left[\begin{array}{ccc}1 & 3 & \frac{1}{3} \\ \frac{1}{3} & 1 & \frac{1}{9} \\ 3 & 9 & 1\end{array}\right]$, he weight of each criterion is (0.2308, 0.0769, 0.6923), accordance proportion $\mathrm{CR}=0<0.1$

Decision matrix from Q2 to $\mathrm{P} 2$ can be expressed as $\left[\begin{array}{ll}1 & 1 \\ 1 & 1\end{array}\right]$, the weight of each criterion is $(0.5,0.5)$ accordance proportion $\mathrm{CR}=0<0.1$

Decision matrix from Q3 to P3 can be expressed as $\left[\begin{array}{ll}1 & 1 \\ 1 & 1\end{array}\right]$, the weight of each criterion is $(0.75$, 0.25)accordance proportion $\mathrm{CR}=0<0.1$

Decision matrix from Q4 to P4 can be expressed as $\left[\begin{array}{cc}1 & \frac{7}{5} \\ \frac{5}{7} & 1\end{array}\right]$, the weight of each criterion is $(0.5833$, 0.4167), accordance proportion $\mathrm{CR}=0<0.1$

The results for accordance of the criteria weight are given in table1, and the overall weights of each layer of criteria weight are given in table 2 .

Table 1. Accordance of the Criteria Weight

\begin{tabular}{|c|c|c|c|c|}
\hline & \multicolumn{3}{|c|}{ Weight } & \multirow{2}{*}{ Accordance Criteria } \\
\cline { 2 - 4 } & $\mathrm{W}_{1}$ & $\mathrm{~W}_{2}$ & $\mathrm{~W}_{3}$ & 0 \\
\hline P1 & 0.2308 & 0.0769 & 0.6923 & 0 \\
\hline P2 & 0.5 & 0.5 & & 0 \\
\hline P3 & 0.75 & 0.25 & & 0 \\
\hline P4 & 0.5833 & 0.4167 & & \\
\hline
\end{tabular}


Table 2. Overall weights of each layer of criteria weight

\begin{tabular}{|c|c|c|c|c|}
\hline & Weight & Criteria Layer & Weight & Overall Weight \\
\hline \multirow{3}{*}{ P1 } & \multirow{2}{*}{0.375} & $\mathrm{Q}_{11}$ & 0.2308 & 0.08655 \\
\cline { 3 - 5 } & & $\mathrm{Q}_{12}$ & 0.0769 & 0.0288375 \\
\cline { 3 - 5 } & \multirow{2}{*}{0.125} & $\mathrm{Q}_{13}$ & 0.6923 & 0.2596125 \\
\cline { 3 - 5 } P2 & \multirow{2}{*}{0.125} & $\mathrm{Q}_{21}$ & 0.5 & 0.0625 \\
\hline \multirow{2}{*}{ P3 } & $\mathrm{Q}_{22}$ & 0.5 & 0.0625 \\
\hline \multirow{2}{*}{ P4 } & \multirow{2}{*}{0.375} & $\mathrm{Q}_{31}$ & 0.75 & 0.09375 \\
\cline { 3 - 5 } & & $\mathrm{Q}_{32}$ & 0.25 & 0.03125 \\
\hline
\end{tabular}

\section{Examples}

A $220 \mathrm{kV}$ oil/water cascaded split transformer is used in this case to demonstrate the algorithm of the proposed model for efficiency evaluation. This split transformer has been operating for thirteen years, defections of the cooling system have been increasing and malfunctions have also occurred during operations. The relative evaluations are listed in table 3. In the meanwhile, the average malfunction period is 300 days and the average reparation time is two days.

Table 3. Parameters of the Target Transformer

\begin{tabular}{|c|c|c|c|}
\hline $\begin{array}{c}\text { Defection(Operation } \\
\text { Data Acquisition \& } \\
\text { analysis) }\end{array}$ & 1.5times/year & $\begin{array}{c}\text { Auxiliary cooling(deploy auxiliary } \\
\text { cooling or not) }\end{array}$ & None \\
\hline $\begin{array}{c}\text { Malfunction(Operation } \\
\text { Data Acquisition) }\end{array}$ & $\begin{array}{c}\text { Malfunction period: } \\
300 \text { days }\end{array}$ & $\begin{array}{c}\text { Heating Point Temperature } \\
\text { rise(Prediction Value, Rated Loads) }\end{array}$ & 56 \\
\hline $\begin{array}{c}\text { Aging(Based on } \\
\text { operation data) }\end{array}$ & $\begin{array}{c}\text { Operational time: } 13 \\
\text { years } \\
\text { Designed operational } \\
\text { time: 25years }\end{array}$ & $\begin{array}{c}\text { Top Layer Oil Temperature } \\
\text { Rise(Measured Value in Reality, Rated } \\
\text { Loads) }\end{array}$ & 20 \\
\hline $\begin{array}{c}\text { Cooling Methods } \\
\text { ODWF }\end{array}$ & $\begin{array}{c}\text { Manufacturer Heating Point } \\
\text { Temperature }\end{array}$ & 60 \\
\hline $\begin{array}{c}\text { Cooling Principles } \\
\text { Oil-Water Cooling }\end{array}$ & $\begin{array}{c}\text { Manufacturer Top Layer Oil } \\
\text { Temperature Rise }\end{array}$ & \\
\hline In service equipment & 8 & & \\
\hline
\end{tabular}

Availability: from expression (3) and (4), there is

$\mathrm{a} 1=\frac{\mathrm{MTBF}}{\mathrm{MTBF}+\mathrm{MTTR}}=0.993$

$\mathrm{a} 2=1-\mathrm{a} 1=0.007$

Establishing Matrix A using a1 and a2. Availability: from expression (6), d11 $=0.99, \mathrm{~d} 12=0.01$

Using Dephi Method and Conventional Evaluation Methods to conduct an overall evaluation of all criteria of this transformer, the results are shown in table 4.

Table 4 Results of the Evaluation

\begin{tabular}{|c|c|c|c|c|c|c|c|c|}
\hline $\mathrm{Q}_{11}$ & $\mathrm{Q}_{12}$ & $\mathrm{Q}_{13}$ & $\mathrm{Q}_{21}$ & $\mathrm{Q}_{22}$ & $\mathrm{Q}_{31}$ & $\mathrm{Q}_{32}$ & $\mathrm{Q}_{41}$ & $\mathrm{Q}_{42}$ \\
\hline 0.80 & 0.76 & 0.26 & 0.90 & 0.85 & 0.72 & 0.32 & 0.81 & 0.88 \\
\hline
\end{tabular}

Using an overall weighed quality coefficients, resulted in the capability coefficient of 0.4381 , and the efficiency can be acquired as,

$$
\mathrm{E}=\mathrm{ADC}=\left[\begin{array}{ll}
0.997 & 0.003
\end{array}\right]\left[\begin{array}{cc}
0.99 & 0.01 \\
0 & 1
\end{array}\right]\left[\begin{array}{c}
0.6602 \\
0
\end{array}\right]=0.6516
$$


If the malfunction period is set to 900 days and the reparation time is set to 1 day, also, auxiliary cooling is deployed and other parameters remain unchanged, Q13 will increase by 0.88 and Q32 will increase to 0.92. Recalculate the efficiency, there is,

$$
\mathrm{E}=\mathrm{ADC}=\left[\begin{array}{ll}
0.999 & 0.001
\end{array}\right]\left[\begin{array}{ll}
1 & 0 \\
0 & 1
\end{array}\right]\left[\begin{array}{c}
0.8212 \\
0
\end{array}\right]=0.8204
$$

The result shows that with lowered rate of system malfunction, increased reparation efficiency and deployment of auxiliary cooling, the efficiency of the cooling system will increase by $26 \%$, which enhanced the overall performance.

\section{Conclusion}

This paper has proposed a model for efficiency evaluation of the cooling system of the split transformer. The proposed model has considered system operation characteristics, extracted critical criteria coefficients and using AHP method and Dephi method for the determination of the decision matrix and capability matrix in ADC method. The proposed model is verified through a test of a 220 $\mathrm{kV}$ oil-water cooling transformer, indicating that this model for evaluating efficiency of the cooling system can be practiced in guiding institutions and operation units for their scientific evaluation of cooling effects of the split transformer.

\section{References}

[1]. Bian Hui, Jiang Yimin, Yuan Congbo, etc. (2011) Application of OF/OF/AN Cooling Mode to Urban Underground Substation. Transformer, 48 (8), 43-47.

[2]. Yu Haibo, Shuai Zhifei, Jiang Yimin. (2014) Operation Analysis of Transformer Cooling Modes of Underground Substation in Shanghai Area. Transformer, 51 (11), 42-47.

[3]. Yu Haibo. (2012) The Research on Large Transformer Cooling System Technique in the Underground High Voltage Substation. Shanghai: Shanghai Jiao Tong University.

[4]. Wang Xuan, Tao Yu, Fan Danying. (2016) Research on Effect Valuation to the Complicated Weapon System Based on ADC. Fire Control \& Command Contro, 2, 113-116.

[5]. Wang Xiaodi, Peng xiuyan, Du Jun. (2012) Research on Equipment Efficiency Evaluation of University based on ADC. Computer Engineering and Design, 33 (11), 4333-4337.

[6]. Ji Xiang Jia Wancai, Sun Qingsheng. (2016) Study of Effectiveness Evaluation Based on ADC and APH Model. Information Technology and Informatization, 4, 96-99. 